\title{
EL MARKETING URBANO COMO HERRAMIENTA DE APOYO A LA GESTIÓN DEL TURISMO DE CIUDAD, ESTUDIO DE UN CASO; EL PATRIMONIO INDUSTRIAL
}

\section{Rigoberto Anguiano Aldama ${ }^{1}$}

Responsable del Centro de estudios de marketing y desarrollo urbano CEMADU Instituto tecnológico superior de Cajeme en Cd. Obregón, Sonora, México.

\section{José Armando Pancorbo Sandoval}

Departamento de economía y turismo de la Universidad de Matanzas "Camilo Cienfuegos"; Cuba.

Palabras Claves: marketing urbano, turismo, patrimonio industrial, identidad urbana y memoria colectiva.

Resumen: El desarrollo urbanístico a nivel internacional afronta varios retos entre los que se encuentra la reutilización de antiguos espacios industriarles abandonados o en declive. Generar nuevos usos y lugares a partir de las huellas de la industrialización implica dotar a estos espacios de nuevos valores de carácter patrimonial y una de las herramientas que hacen posible este hecho, es la utilización del Marketing Urbano que posibilita alternativas para la toma de decisiones a los actores locales, quienes deciden qué hacer con estas antiguas instalaciones industriales. El objetivo principal de este artículo es exponer las ventajas de aplicar el marketing urbano para poder definir el empleo más adecuado para estas zonas que tuvieron un gran valor en su momento y que ahora pueden erigirse como parte del patrimonio, identidad y memoria colectiva de una ciudad.

\section{Introducción.}

El territorio ha constituido históricamente una compleja estructura socioeconómica que reúne potencialmente las características de un bien cultural. Los territorios marcados por procesos industriales son testigos poderosos e insustituibles de importantes transformaciones socioeconómicas y políticas que han creado los atributos de una determinada localidad en determinados momentos de su historia y evolución, como puede ser el caso de la ciudad de Bilbao, España. Dicha ciudad, durante años tuvo una marcada vocación industrial y en este momento apuesta por ser una ciudad de servicios y estrechamente vinculada al turismo, pero siempre respetando su pasado industrial.

Este doble motivo de presencia, como soporte físico y como producto histórico-geográfico, hace de la aproximación territorial una dimensión inseparable de cualquier reflexión sobre el patrimonio industrial y, desde luego, de todo intento por preservarlo, difundirlo y utilizarlo.

\footnotetext{
${ }^{1}$ Persona de contacto MA. Rigoberto Anguiano Aldama, correo: ranguiano@itesca.edu.mx
} 
En el proceso de industrialización ha jugado y juega un rol vital la arquitectura como la disciplina que va a permitir crear la estructura que contendrá la tecnología industrial en cuestión y que además va a materializar todos los avances científicos y tecnológicos en términos de la concepción de nuevas tipologias con lenguaje propio, introducción de nuevos materiales constructivos y nuevas técnicas de construcción.

Uno de los temas que sobre esta arquitectura ha suscitado más debate últimamente, es la intervención al patrimonio industrial, para transformarlo en parte de los espacios urbanos atractivos para los residentes y para los visitantes.

En definitiva, la cuestión es cómo lograr crear un producto turístico atractivo, que posibilite favorecer el posicionamiento nacional e internacional de una ciudad como destino de turístico, recreativo y de negocios, a partir de esta herencia acumulada en años por su actividad productiva.

"En nuestras ciudades casi no sería necesario construir nada nuevo: hay en ellas arquitectura suficiente para incluir todas las necesidades posibles, si queremos conservar todo cuanto es testimonio utilizable de la historia. Hay arquitectura suficiente para la residencia, para los museos, para los servicios colectivos, para las escuelas [...] e incluso para regenerar los lugares de producción -las fábricas, ¿por qué no?-y, por tanto, para conseguir la homogeneización social y funcional de la ciudad." Oriol Bohigas (1986)

Contradictoriamente, los intereses de las empresas de la región, al igual que los de los residentes y visitantes de la ciudad, no son siempre las mismas, existen en todos ellos demandas de nuevos espacios, para poder satisfacer necesidades recreativas, inmobiliarias, de inversión etc.

Por lo tanto, se justifica que las autoridades locales intervengan los espacios industriales, obsoletos u abandonados, para crear espacio a nuevas colonias residenciales, plantas industriales o de servicios.

Es precisamente en este punto donde se debe evaluar y determinar lo más útil para la ciudad: ¿destruir el patrimonio industrial?, o ¿conservarlo para la posteridad, como parte de la oferta urbana de la localidad en cuestión? Pero para poder dar respuesta a estas interrogantes se debe comprender ante todo el concepto de patrimonio industrial. Un ejemplo interesante de lo que este tipo de patrimonio puede significar en términos de desarrollo para una ciudad, es el caso de la zona agro industrial en Ciudad Obregón, Sonora.

Ciudad fundada en 1928 en las planicies desérticas de la costa de Sonora, que en su momento represento la conquista del territorio en base de la voluntad del hombre y el desarrollo de la tecnología agraria y que hoy requiere de referencias identitarias que den sustento a una sociedad que ha diversificado su base productiva, a expandido sus limites urbanos y busca proyectarse al futuro, sin olvidar las huellas de un pasado inmediato y los elementos que conforman su memoria colectiva (Figuras 1,2 y 3 ) 


\section{Architecture, City, and Environment
Arquitectura, Ciudad y Entorno}
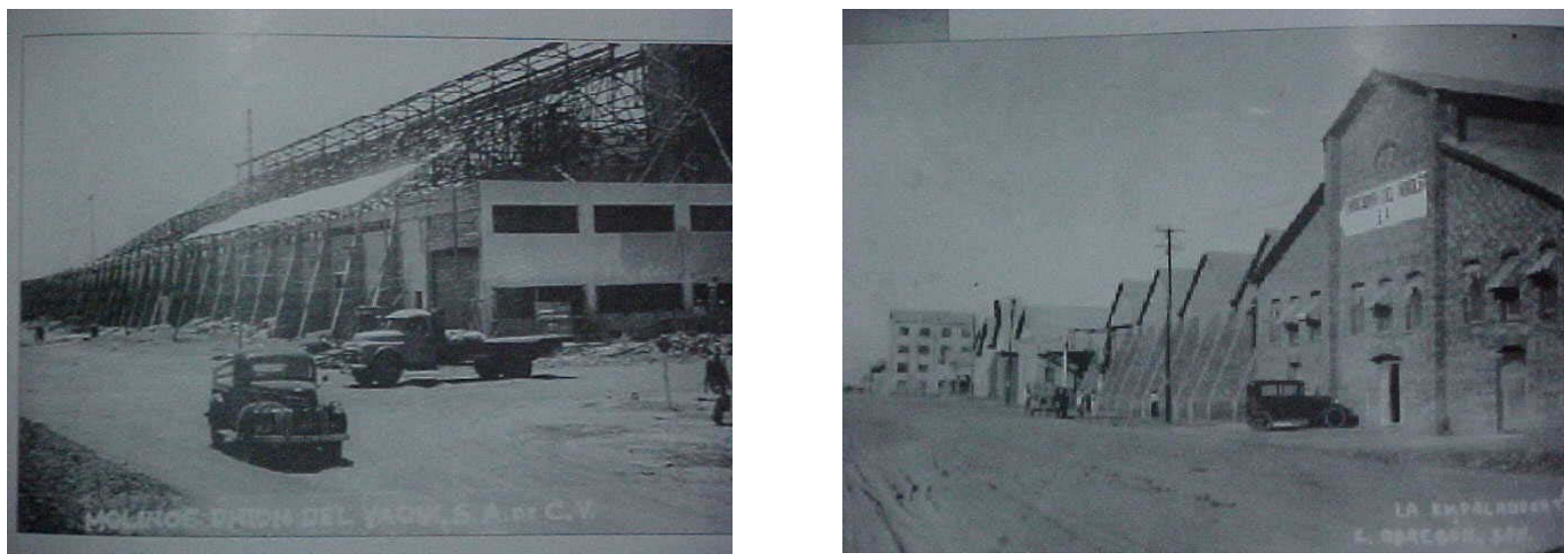

Figura 1, Figura 2: Construcción de almacenes de granos en Ciudad Obregón 1932

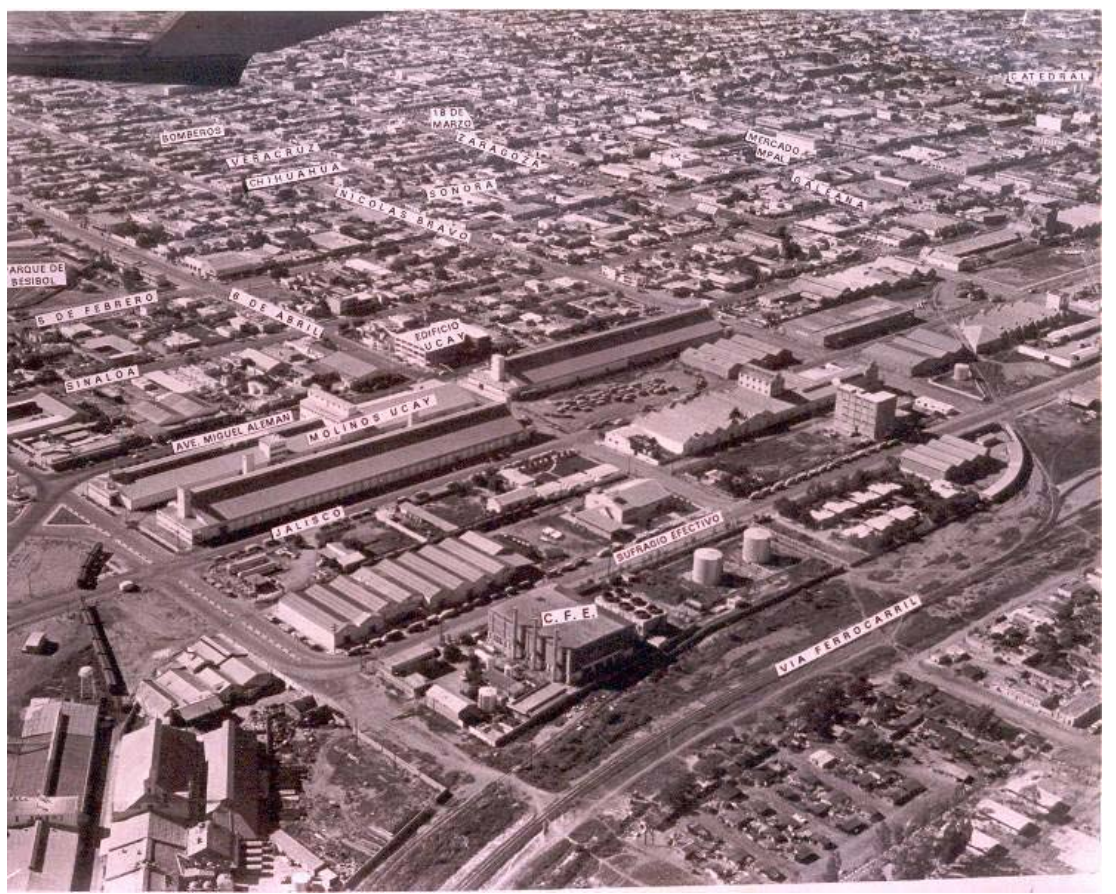

Figura 3: Vista panorámica de zona industrial de Ciudad Obregón 1965 


\section{El patrimonio industrial y su puesta en valor como estrategia de desarrollo en la ciudad}

El término se comienza a utilizar a partir de una publicación de Michael Rix titulada "El Historiador Amateur" en el año 1955, donde se enfatiza la importancia de la conservación de los testimonios heredados de la revolución industrial, tanto por el valor histórico que poseen como por su importancia en la formación de la identidad de los grupos sociales involucrados. A partir de esta fecha, y durante las décadas del 50 y del 60' se comenzarían con los primeros estudios sobre arqueología industrial en Inglaterra, Estados Unidos y otros países, que han continuado en la actualidad en diversas partes del mundo; y con estos estudios e investigaciones se comenzaron a generar los primeros intentos de recuperación y revaloralización de los espacios industriales abandonados o subaprovechados, transformándolos en museos, incorporándolos a circuitos turísticos por ejemplo.

A lo largo del tiempo algunos lugares han encontrado en su herencia industrial elementos patrimoniales que se han valorado en el mercado, desde el punto de vista arquitectónico, museístico o recreativo. Existen ya un suficiente número de iniciativas y experiencias respecto a la utilización de los restos industriales del pasado y actuales. Como centros de recursos de carácter cultural y didáctico, las cuales pueden servir de referencia para iniciativas semejantes en nuestra ciudad. De la utilización del patrimonio industrial, como atractivo de las ciudades para visitantes extranjeros, se pueden citar varios casos como por ejemplo, el Museo de la Electricidad de Lisboa, el Museo de la Ciencia y la Tecnología de Cataluña, en Terrassa Provincia de Barcelona, España. el Parque fundidora en Monterrey, México o Puerto Madero en Buenos Aires, Argentina, además de otros países como Inglaterra, Francia, Alemania, Bélgica y Estados unidos que han rescatado y regenerado muchas zonas fabriles y portuarias, para dedicarlas luego al turismo y convertirlas en referencias importantes para sus ciudades.

Algunos de estos proyectos se incluyen dentro de los movimientos de dinamización territorial que en las últimas décadas proponen que es posible considerar que los recursos del turismo, el ocio, la promoción cultural, natural y de cualquier tipo de atractivo con un cierto interés, integran un potencial estratégico de futuro en algunas áreas del interior: Se contemplan estos recursos como una alternativa para un nuevo modelo de desarrollo local que ayude a superar un periodo de recesión económica, demográfica y social, consecuencia de la desaparición o el declive irreversible de las actividades productivas tradicionales, y de su no- sustitución por otras en breve" (Llurdes i Coit, 1995).

En México, lamentablemente aún en ocasiones se superpone la obsolescencia funcional que suponen estos espacios que fueron diseñados específicamente para otro uso y los extensos metros cuadrados sin uso específico, que afectan la gestión empresarial, no existiendo en ocasiones un inventario evaluado por especialistas en el sector de la arquitectura y arqueología industrial sobre la importancia de las edificaciones, maquinas y componentes. Diagnosticar si se pueden considerar dentro de los parámetros de Patrimonio Industrial. De tal modo autores como la especialista María Bustamante Harfush ${ }^{2}$, cita el caso en Tultitlán, Estado de México, de la embotelladora Bacardí, localizada sobre la autopista México-Querétaro, proyecto de las oficinas del afamado arquitecto Ludwig Mies van der Rohe y del proyecto de la planta embotelladora que estuvo a cargo de Félix Candela. Ninguna de estas construcciones, según la experta Bustamante, está catalogada por las instituciones encargadas de hacerlo; sin embargo, sí están catalogadas dentro de algunas guías de arquitectura del Siglo XX.

2 Para más detalle se recomienda leer el artículo titulado La reciente demolición de la fábrica Chrysler de México de dicha autora. 
Casos como los citados anteriormente, indican la necesidad de conciliar los elementos técnicos, históricos y sociales antes de decidir la demolición de una infraestructura que ha cerrado su ciclo productivo, pero que puede iniciar otro vinculado a la gestión de servicios o inclusive mantenerse como un museo donde se explique como funcionaban algunas tecnologías obsoletas en este momento.

Pero para poder demostrar la necesidad de mantener una edificación como puede ser antiguos talleres de ferrocarril o una planta eléctrica, es imprescindible demostrar la viabilidad económica y social del nuevo uso para los residentes, empresarios y demás implicados en la gestión de una ciudad.

Es importante considerar a la edificación a evaluar dentro de un destino, donde su nuevo uso, posibilitará mejorar la calidad de vida de los residentes en la misma. Es necesario desarrollar estudios de mercado que posibiliten determinar la factibilidad de la instalación como sede de nuevas actividades que pueden ser sociales, económicas o administrativas. Es en este momento en que se hace válida la utilización de nuevas técnicas como es el Marketing Urbano.

\section{Marketing Urbano como estrategia para revitalizar el patrimonio industrial.}

El Marketing Urbano como subsistema del sistema del Marketing, va a estudiar el comportamiento de las ciudades a partir de sus necesidades, pero también de los deseos de cómo quieren que se desarrollen los ciudadanos y demás consumidores de la misma (inversores, turistas, entre otros).

El Marketing Urbano posibilita concretar pues, la planificación estratégica territorial en forma tal, de lograr entre otros los siguientes beneficios (Godet, 1991; Portas, 1996; Rabinovitch y Leitman, 1996):

$x$ Ofrece una visión global e intersectorial del sistema urbano a largo plazo.

$x$ Identifica tendencias y anticipa oportunidades.

$x$ Formula objetivos prioritarios y concentra recursos limitados en temas críticos.

x Estimula la comunicación entre los diferentes agentes locales y el entorno exterior.

$\times \quad$ Favorece al tejido social y la conservación del medio ambiente.

$\times$ Implica a la iniciativa privada.

x Amplía la perspectiva política y social.

x Favorece el establecimiento de alianzas entre diversas entidades y fuerzas políticas.

El Marketing Urbano tiene la responsabilidad de facilitar el proceso de intercambio que la ciudad establece con su mercado. La gestión del proceso de intercambio supone, por una parte, el diseño y la realización de actividades orientadas a investigar las necesidades y deseos del mercado, con la finalidad posterior de diseñar una oferta que se adapte a tales exigencias. Asimismo, y a posteriori, se trata de investigar también cuál es el grado de satisfacción que los clientes alcanzan con los productos o servicios ofertados (seguridad ciudadana, salud, educación, turismo, etc.) y por otra parte implica el desarrollo de actividades tendentes a despertar la demanda y a servir o colocar los productos o servicios en el mercado considerado.

En definitiva, es la orientación de marketing urbano la que conecta la ciudad con su mercado, investigando y atendiendo las necesidades que en el mismo se manifiestan; y todo esto se realiza en competencia con otras ciudades que actúan en el mismo mercado. 
Uno de los principales problemas con que se enfrentan hoy en día los gestores urbanos es la reutilización de espacios industriales que han sido relevados por las nuevas tecnologías. En este sentido los expertos en marketing sugieren desde hace varios años la reconversión de este patrimonio industrial en espacios tematizados que den respuesta a las necesidades y deseos de los ciudadanos y demás demandantes del producto urbano.

\section{La tematización y los riesgos de la exclusión urbana a partir del uso de las herramientas del marketing de ciudad.}

La tematización de las ciudades se enmarca dentro de las estrategias de posicionamiento de éstas en un ambiente de creciente competitividad donde el ocio juega un papel fundamental. La cultura (y evidentemente el patrimonio cultural y la creación artística) juega aquí un rol diferenciador en el marco de la configuración de las políticas urbanas ya que se pueden constituir como motor económico de desarrollo.

Antes de centrarnos en el caso concreto de la tematización de las ciudades a partir de sus recursos culturales es preciso contextualizar el proceso actual en relación con el uso de diferentes estrategias para incorporar el ocio en sus políticas de regeneración urbana. Es preciso referirnos al concepto de "ciudad emprendedora" que, como sugiere Sara González (2002), "viene desde hace algunos años aglutinando una corriente de estudios provenientes sobre todo de la geografía urbana y que hace referencia a las ciudades que adoptan estrategias más propias de la empresa que de los poderes públicos para salir adelante y tener éxito en una economía cada vez más global". La "ciudad emprendedora" viene asociada a "nuevas políticas urbanas", término empleado por Cox (1993) para bautizar a los estudios urbanos centrados especialmente en profundizar sobre las conexiones entre las ciudades y el espacio económico global. La diferenciación y los productos de valor añadido se tornan fundamentales para determinar las ventajas competitivas entre las ciudades, especialmente aquellas ciudades industriales que han perdido competitividad y están desarrollando nuevos emprendimientos de reconversión industrial y una orientación hacia el sector servicios.

La necesidad o la creciente tendencia a utilizar estrategias empresariales como las campañas de marketing en las ciudades, está directamente relacionada con la competitividad interurbana.

En este sentido no sólo se emplean estas estrategias para favorecer la instalación de nuevas empresas mediante condicionantes de tipo económico y tecnológico sino que también se aplican en la diferenciación y diversificación de ciudades como sedes de grandes eventos, como destinos para el estudio universitario o aprendizaje de idiomas o como marcas turísticas internacionales.

Se han empezado a explorar los diferentes gustos estéticos y se ha otorgado más importancia a la producción y consumo de lo que Bourdieu (2007) llama el "capital simbólico". En el contexto urbano, esto se ha reflejado en la mayor demanda por parte de las crecientes clases medias de equipamientos de ocio e instalaciones culturales, que hay que interpretar como consecuencia de las políticas culturales públicas de los últimos veinte años que tenían como objetivo favorecer el acceso a la cultura a la ciudadanía, creando e impulsando pautas de consumo cultural, y educando públicos que demandan cada vez más servicios de mayor calidad (Jensen- Butler 1997). 
La tematización patrimonial de las ciudades responde a las expectativas ocasionadas por el turismo cultural, una de las modalidades más dinámicas establecidas recientemente desde el punto de vista de la demanda. No son pocas las ciudades que han desarrollado políticas, programas y acciones para (re)valorizar sus propios recursos para configurar productos turísticos comercializables tanto en el mercado de proximidad como en el nacional e internacional. De todas formas, nos sumamos a la sugerencia de Andre, M. (2003), que consideran que "la actividad turística no debe contemplarse como una finalidad en sí misma, sino como un instrumento que permita el desarrollo óptimo de la actividad económica y social del territorio en cuestión". Se hace necesario definir los destinos turísticos de forma integral, es decir, mediante la incorporación de todos los recursos y/o productos existentes a una oferta global, articulada en el territorio y acorde al tejido social y económico presente en el mismo.

El Marketing Urbano tiene entre sus objetivos, modificar la imagen de la ciudad, como es el caso de Bilbao, de ciudad industrial-siderurgica a ciudad de servicios. Estas estrategias no sólo van asociadas al exterior para posicionar la ciudad en el "mercado de localizaciones" o como ciudad de negocios, eventos o destino turístico. Las acciones hacia los residentes van destinadas a promocionar la ciudad en relación con parámetros vinculados con el bienestar económico y la calidad de vida. La cultura se integra en las estrategias de ofrecer una imagen de ciudad post-industrial y post-moderna que se reflejan en la construcción de un nuevo paisaje urbano como es el caso de la ría de Bilbao con el Museo Guggenheim, obra de Frank O. Gehry. Del mismo modo que Frank Lloyd Wright con su museo Guggenheim de New York en forma de espiral, Gehry creó un espacio para el arte que se convirtió al mismo tiempo en una escultura gigante de titanio, icono de la ciudad y referencia mundial de los nuevos tiempos en la arquitectura.

Una de las estrategias que están desarrollando la mayor parte de las ciudades es el uso de la tematización o de la multitematización de su espacio para la presentación de productos de turismo cultural orientados tanto al turismo de proximidad como al turismo internacional. El patrimonio cultural suele ser la base sobre la que se estructuran la mayor parte de propuestas (Ballart y Tresserras 2001).

El patrimonio industrial y otros recursos culturales pueden constituir una auténtica variable de desarrollo. "La gestión inteligente del patrimonio está suponiendo en diversos lugares uno de los factores claves para su desarrollo económico, porque atrae turismo e inversiones, porque genera actividades y lugares de trabajo y, fundamentalmente, porque refuerza la autoestima de la comunidad" (Sabaté y Lista, 2001)

La revitalización del patrimonio industrial privilegia la construcción de una imagen que otorga identidad al territorio, donde el patrimonio y otros recursos culturales, históricos y escénicos se combinan, exponen, aumentan y promueven intencionadamente para formar un paisaje acordado, convenido que cuenta la historia de dicho territorio y sus residentes. El patrimonio industrial requiere e inicia un cambio en los significados simbólicos de un determinado paisaje o territorio. Transforman áreas de decadencia productiva tanto por el fomento del turismo, como por la revitalización del espíritu comunitario, los cuales conjuntamente pueden atraer nuevas inversiones.

La imagen de estos proyectos generalmente es desarrollada alrededor un tema común, de episodios productivos o históricos que han influido en la cultura y la historia de un territorio. Por ejemplo, los modelos seguidos en la gran mayoría de los parques patrimoniales desarrollados en Estados Unidos realzan la sustancia histórica y la identidad geográfica (muchos proyectos se asocian con un recurso natural o cultural de gran escala) como ingredientes fundamentales en la composición de la imagen de un parque patrimonial. 
Uno de los errores mas comunes hoy en día en la planificación territorial es desarrollar lo que denominan algunos autores como la "mcdonalización" de la imagen urbana, en este sentido algunas antiguas instalaciones industriales son reconvertidas en zonas comerciales y/o de ocio en general, sin que se respeten los elementos diferenciadores de estas instalaciones y que significan para los ciudadanos referencias emocionales importantes, lo que supone un tratamiento maduro y consensuado entre técnicos, autoridades y ciudadanos. En definitiva intervenir estos edificios industriales sugiere una política pública de alta consideración a su estatus patrimonial, pero también un ánimo de generar espacios públicos altamente redituables en donde sea posible generar condiciones ventajosas para la calidad de vida de los habitantes y evitar en lo posible que se conviertan en espacios de exclusión. En sociedades abiertas y participativas los proyectos de reconversión del patrimonio industrial generan sinergias muy importantes y se encuentran en la mesa de discusión. Horacio Cappel (2007) La conservación del patrimonio industrial es importante para entender el presente y para proyectar el futuro. Para evitar la homogenización. Por que con su destrucción se pierde diferencia, especificidad y diversidad: Por que sus muestras constituyen alternativas al modelo urbano uniformizador y posibilidades diversas para la vida en la ciudad, condición para la supervivencia de la multiplicidad urbana.

\section{Conclusiones:}

5.1 El patrimonio industrial y el paisaje cultural constituyen elementos dinamizadores del desarrollo territorial. De modo especial el papel de catalizadores en la reactivación de paisajes y áreas que buscan superar los impactos territoriales, económicos y sociales, derivados de procesos de pérdida de base económica y/o des-industrialización.

5.2 El rescate de la patrimonio industrial debe partir de una evaluación técnica realizada por expertos en arquitectura en colaboración con especialistas en cultura, economía y administrativos, para poder determinar no sólo la valoración técnica sino también la evaluación hedónica de la instalación.

5.3 La aplicación de las técnicas de marketing urbano, facilitará la revitalización de las ciudades en crisis mediante el desarrollo del turismo y en especial cómo podrían convertirse las instalaciones industriales obsoletas a los usos turísticos.

5.4 El turismo y el ocio como actividades económicas posibilitan recuperar espacios abandonados en centros cívicos y/o turísticos, pero para ello se requiere de un serio trabajo de equipo de arquitectos que posibiliten salvar los valores de la edificación como parte de sus atributos como nuevo producto turístico, como puede ser estilo arquitectónico, materiales constructivos, tecnologías empleadas para la construcción, etc.

5.5 El desafío a que se enfrentan los equipos de arquitectos en este momento en la revitalización del patrimonio industrial es en primer lugar demostrar la validez de la infraestructura como patrimonio industrial y en segundo lugar dar respuestas técnicas a su recuperación, para lo cual su alianza con los expertos en marketing urbano, les posibilitará determinar oportunidades en el mercado del ocio y del turismo. 


\section{Bibliografía.}

ANDRE, M., Cortes I. y López J. (2003) "Turismo cultural: Cuando el recurso cultural supera al destino turístico. El caso de Figueres". XII simposio internacional de turismo y ocio (Barcelona abril 2003). ESADE-Fira de Barcelona. Barcelona

BALLART, Hernández, J. y TRESSERRAS, J. (2001). Gestión del patrimonio cultural. Editorial Ariel. Barcelona. España

BOURDIEU, P. (2007) "Capital cultural, escuela y espacio social” Siglo XXI editores. Págs. 2340. México

CAPEL, H. (1996): «La rehabilitación y el uso del patrimonio histórico industrial». Document d’Análisi Geografica, $n^{\circ} 29$, pp. 19-50.

COX, K.R. (1993) "The local and the global and the new urban politics: a critical review" .Environment and planning: Society and Space D.N 11 Pags. 433-448

GODET, M. (1991) Prospectiva y planificación estratégica. S.G. Editores. 346 Pp. Barcelona

GONZALEZ, Sara. http://www.ciudad-derechos.org/espanol/invest.html

JENSEN-BUTTLER, C y VAN WESSEP, J., (1997) "Perspective: Competition, urban planning and urban policy" en JENSEN-BUTTLER, C. SACHAR, A. Y VAN WESSEP, J. (eds.) European cities in competition, Averbury, Aldershot

JONAS, Rabinovith y JOSEF, Leitman. (1996) Revista Investigación y ciencia, ISSN 0210136X, pags. 62-69 Universidad de la Rioja, España

LLURDES I COIT, Joan. (1995). EI Turismo de Patrimonio Industrial y Minero. Una experiencia de turismo interior explotada en el Estado español. Departamento de Geografía. Universidad Autónoma de Barcelona. España.

PORTAS, Nuno. El planeamiento urbano como proceso de regulación variable", en Revista Ciudades $N^{\circ} 3$, Instituto de Urbanística de la Universidad de Valladolid, España, 1996.

SABATÉ, J. y LISTA, A. (2001) Casos d'estudi europeus. En: SABATÉ, J y SCHUSTER, J M (eds.) Projectant l'eix del Llobregat. Paisatge cultural i desenvolupament regional, Barcelona 
